expressed (ref. 21 and M. Parsons et al., personal communication). In addition, the mini-exon sequence (or a conserved homologue) has been shown to be present in the genomes of several related species and genera which lack antigenic variation of the type observed for T. brucei $i^{22}$.

Could discontinuous transcription be operating in these other cases? This question cannot, as yet, be answered, but some precedent does exist in systems which are otherwise totally unrelated. Transcription of the influenza virus is known to require a 5 -capped primer (10-15 nucleotides long) derived from cleavage of host mRNAs ${ }^{36,37}$. Coronavirus transcripts, on the other hand, are known to have a common 5 '-leader sequence which is seemingly not joined to the coding portion of the RNA by conventional splicing, again strongly suggesting discontinuous transcription ${ }^{38,39}$. The fact that, in both these cases, transcription occurs by an RNA-dependent viral transcriptase may be an important distinction but they do provide a mechanistic precedent for discontinuity in primary transcripts. The observation made in Fig. 6 that a snRNA of yeast has substantial homology with the region known to be a donor splice site in medRNA, suggests that this snRNA (of unknown function) might be similarly involved in discontinuous transcription, albeit on a lesser scale ( $\mathrm{snR} 3$ is nonessential; ref. 32 ). Such activity could further provide a molecular basis for the long-standing observation that heterogeneous nuclear RNAs often possess sequences at their 5 -ends which are derived from middle repetitive DNA ${ }^{40-42}$ and that such sequences might be important in the control of developmentally regulated gene expression. The testing of this interesting hypothesis in trypanosomes may now be possible.

We thank Mr M. van Bree for technical assistance; Drs N. Agabian, P. Borst, G. Cross, J. Donelson, M. Lai, K. Stuart and their colleagues for exchange of information before publication; Dr M. Fedor for producing the synthetic oligonucleotide; and Ms M. A. Siri for secretarial help. This work was supported in part by a grant from the American Cancer Society (ACS IN 32).

Received 22 March; accepted 22 June 1984.

1. Cross, G. A. M., Holder, A. A., Allen, G. A. \& Boothroyd, J. C. Am. J. trop. Med. Hyg. 29(5) Suppl., 393-417 (1980).

2. Englund, P. T., Hajduk, S. L. \& Narini, J. C. A. Rev. Biochem. 51, 695-726 (1982).

3. Borst, P. \& Cross, G. A. M. Cell 29, 291-303 (1982).

4. Pays, E., Lheureux, M. \& Steinert, M. Nature 292, 265-267 (1981).

5. Hoeijmakers, J. H. J., Borst, P., Van den Burg, J., Weissmann, C. \& Cross, G. A. M. Gene 8, 391-417 (1980).

6. Van der Ploeg, L. H. T. et al. Nucleic Acids Res. 10, 593-609 (1982).

7. Capbern, A. Giroud, C. Baltz, T. \& Mattern, P. Expl Parasit. 42, 6-13 (1977).

8. Cross, G. A. M. Parasitology 71, 393-417 (1975)

9. Hoeijmakers, J. H. J., Frasch, A. C. C., Bernards, A., Borst, P. \& Cross, G. A. M. Nature 284, 78-80 (1980).

10. Pays, E., Van Meirvenne, N., Le Ray, D. \& Steinert, M. Proc, natn. Acad. Sci. U.S.A. 78, 2673-2677 (1981)

11. Williams, R. O., Marcu, K. B. \& Majiwa, P. A. O. Nature 282, 847-849 (1979).

12. Van der Ploeg, L. H. T. et al. Nucleic Acids Res. 10, 3591-3604 (1982).

13. Boothroyd, J. C. \& Cross, G. A. M. Gene 20, 281-289 (1982).

13. Loothroyd, J. C. \& Cross, G. A. M. Gene 20, 281-289 (1982).

14. Longacre, S. et al. Molec. cell. Biochem. 3,
15. Bernards, A, et al. Cell 27, 497-505 (1981).

16. Van der Ploeg, L. H. T., Bernards, A., Rijsewijk, F. A. M. \& Borst, P. Nucleic Acids Res. 10, 593-609 (1982).

17. Michiels, F. et al. EMBO J. 2, 1185-1192 (1983).

18. Liu, A. Y. C., van der Ploeg, L. H. T., Rijsewijk, F. A. M. \& Borst, P. J. molec. Biol. 167, 57-75 (1983).

19. Campbell, D. A., van Bree, M. \& Boothroyd, J. C. Nucleic Acids Res. 12, 2759-2774 (1984).

20. Nelson, R. G. et al. Cell 34, 901-909 (1983)

21. De Lange, T. et al Cell 34,891-900 (1983).

22. Nelson, R. G. et al. Nature 308, 665-667 (1984).

23. Borst, P. et al. Eur. J. Biochem. 137, 383-389 (1983)

24. McDonell, M. W., Simon, M. N. \& Studier, F. W. J. molec. Biol. 110, 119-146 (1977),

25. Bolivar, F. et al. Gene 2, 95-113 (1977).

26. Rigby, P. W. J., Diekmann, M., Rhodes, C. \& Berg, P. J. molec. Biol. 113, 237-251 (1977).

27. Grunstein, M. \& Hogness, D. S. Proc. natn. Acad. Sci. U.S.A. 72, 3961-3965 (1975).

28. Twigg, A. J. \& Sherratt, D. Nature 283, 216-218 (1980).

29. Berk, A. J. \& Sharp, P. A. Cell 12, 721-732 (1977).

30. Maniatis, T., Fritsch, E. F. \& Sambrook, J. Molecular Cloning. A Laboratory Manual (Cold Spring Harbor Laboratory, New York, 1982).

31. Thomas, P. S. Proc. natn. Acad. Sci. U.S.A. 77, 5201-5205 (1980).

32. Tollervey, D., Wise, J. A. \& Guthrie, C. Cell 35, 753-762 (1983).

33. Cordingley, J. S. \& Turner, M. J. Molec, biochem. Parasit. 1, 91-96 (1980)

34. Breathnach, R. \& Chambon, P. A. Rev. Biochem. 50, 349-383 (1981).

35. Wise, J. A. et al. Cell 35, 243-751 (1983).

36. Plotch, S. H. Bouloy, M. Ulmanen, I \& Kug, R. M. Cell 23, 847-858 (1981)

37. Lamb, R. A. \& Choppin, P. W. A. Rev. Biochem. 52, 467-506 (1983).

38. Siddell, S., Wege, H. \& ter Meulen, V. J. gen. Virol. 64, 761-776 (1983).

39. Baric, R. S., Stohlman, S. A. \& Lai, M. M. C. J. Virol. 48, 633-640 (1983)

40. Firtel, R. A. \& Lodish, H. F. J. molec. Biol. 79, 295-314 (1973).

41. Zuker, C. \& Lodish, H. F. Proc. natn. Acad. Sci. U.S.A. 78, 5386-5390 (1981)

42. Davidson, E. H. \& Posakony, J. W. Nature 297, 633-635 (1982).

43. Maxam, A. M. \& Gilbert, W. Meth. Enzym 65, 499-560 (1980).

44. Sanger, F. \& Coulson, A. R. FEBS Lett. 87, 107-110 (1978).

\title{
LETTERSTONATURE
}

\section{On the origin of Triton and Pluto}

\section{William B. McKinnon}

Department of Earth and Planetary Sciences, and McDonnell Center for the Space Sciences, Washington University, Saint Louis, Missouri 63130, USA

Lyttleton hypothesized long ago that Triton and Pluto originated as adjacent prograde satellites of Neptune ${ }^{1}$. With the presently accepted masses of Triton and Pluto-Charon ${ }^{2,3}$, however, the momentum and energy exchange that would be required to set Triton on a retrograde trajectory is impossible. The mass of Triton has probably been seriously overestimated ${ }^{4,5}$, but not by enough to relax this restriction. It is implausible that the present angular momentum state of Pluto-Charon has been significantly influenced by Neptune ${ }^{6}$. It could not acquire such angular momentum during an ejection event unless a physical collision was involved, which is quite unlikely. The simplest hypothesis is that Triton and Pluto are independent representatives of large outer Solar System planetesimals. Triton is simply captured, with potentially spectacular consequences that include runaway melting of interior ices and release to the surface of clathrated $\mathrm{CH}_{4}, \mathrm{CO}$ and $\mathrm{N}_{2}$ (ref. 7). Condensed remnants of this proto-atmosphere could account for features in Triton's unique spectrum ${ }^{8-11}$.

The dynamics of Triton's orbital evolution are considerably simplified by the fact that its specific dissipation function, $Q$, at tidal frequencies, is much less than that of Neptune $\left(Q_{T} \ll Q_{N}\right)$. Here $I$ assume a standard solid-body $Q$ for Triton of $\sim 100$ (ref. 12). A lower bound on $Q_{N}$ can be derived by requiring that the outward orbital evolution of a satellite given by

$$
\frac{\mathrm{d} a_{\mathrm{s}}}{\mathrm{d} t}=3 k_{2 \mathrm{~N}} \sqrt{\frac{G}{m_{\mathrm{N}}}} \frac{R_{\mathrm{s}}^{5} m_{\mathrm{s}}}{Q_{\mathrm{N}} a_{\mathrm{s}}^{11 / 2}}
$$

is not so rapid that the satellite originated at the corotation radius of Neptune 4,500 $\mathrm{Myr}$ ago $^{12}$ (where $m_{\mathrm{s}}$ and $a_{\mathrm{s}}$ are the satellite's mass and semimajor axis; $m_{\mathrm{N}}, R_{\mathrm{N}}$ and $k_{2 \mathrm{~N}}$ are Neptune's mass, radius, and tidal-effective potential Love number of the second degree; and $G$ is the gravitational constant). $k_{2 \mathrm{~N}}$ is estimated at 0.43 , subject to uncertainties in Neptune's rotation rate and $J_{2}$ (the coefficient of the second harmonic of the gravitational potential) (see ref. 13); other parameter values are given in Table 1 . If Neptune's third satellite ${ }^{14}$ is confirmed and proves to be regular and non-commensurate, then $Q_{N} \geqslant 10^{4}$. A lower bound on the $Q$ of Uranus of $\sim 2 \times 10^{4}$ is set using Miranda ${ }^{15}$ and a $k_{2}$ for Uranus of 0.28 . The $Q$ s of both planets should be comparable, and are probably much larger.

Accordingly, the monthly radial tide raised on Triton by Neptune dominates Triton's orbital evolution, except for orbits of very small eccentricity. This tide does not transfer angular momentum for a synchronously-rotating Triton, but in the cases of interest here, such non-synchronous spin angular momentum would be negligible compared with orbital angular momentum. The present fractional rate of change in Triton's orbital angular momentum, due to the tide raised on Neptune by Triton, is 
( $\left.\mathrm{d} a_{\mathrm{T}} / \mathrm{d} t\right) 2 a_{\mathrm{T}}$, where $a_{\mathrm{T}}$ is Triton's semimajor axis. Using equation (1), the total change over the age of the Solar System is a few per cent for $Q_{N} \sim 10^{5}$. Changes in inclination are similarly small.

Lyttleton's hypothesis is evaluated as follows: Triton and Pluto originate as regular direct satellites of Neptune, in circular orbits; orbital evolution brings them into a range of significant gravitational interaction, say, within the sphere of influence of the more massive satellite (the radius of which is given by $s \simeq a_{>}\left(m_{>} / m_{\mathrm{N}}\right)^{2 / 5}$ (ref. 16), where $a_{>}$and $m_{>}$are the semimajor axis and mass of the more massive satellite), and Triton is sent into a retrograde orbit, potentially elliptical, with the same angular momentum as it has today. Conservation of angular momentum requires that the angular momentum of Pluto, $l_{\mathrm{P}}$, increases to

$l_{\mathrm{P}}=\left(G m_{\mathrm{N}}\right)^{1 / 2}\left\{\left(\alpha m_{\mathrm{T}} \sqrt{a_{\mathrm{T}}}+m_{\mathrm{T}} \sqrt{a}+m_{\mathrm{P}} \sqrt{a}\right)^{2}+\beta^{2} m_{\mathrm{T}}^{2} a_{\mathrm{T}}\right\}^{1 / 2}$

where $m_{\mathrm{T}}$ and $m_{\mathrm{P}}$ are the masses of Triton and Pluto-Charon, $\alpha$ and $\beta$ are $\cos (21.5)$ and $\sin (21.5)$-accounting for Triton's inclination of $158.5^{\circ}$ (ref. 17), and $a$ is the semimajor axis of the interaction. Pluto's post-encounter orbital energy is minimized for velocities perpendicular to its radius vector. The semimajor axis (elliptic or hyperbolic) of the new orbit, with respect to Neptune, is then:

$$
a_{\mathrm{P}}=\left|\left(l_{\mathrm{P}}^{2} / G m_{\mathrm{N}} m_{\mathrm{P}}^{2} a\right)-2\right|^{-1} a
$$

If $l_{\mathrm{P}}>\left(2 G m_{\mathrm{N}} a\right)^{1 / 2} m_{\mathrm{P}}$, the orbit will be hyperbolic. This is guaranteed for $m_{\mathrm{T}}>(\sqrt{2}-1) m_{\mathrm{P}}$.

The relationship (3) is illustrated in Fig. 1 for a range of plausible mass ratios $m_{\mathrm{T}} / m_{\mathrm{P}}$. All the $a_{\mathrm{P}}$ shown correspond to hyperbolic trajectories. Orbits with $a_{\mathrm{P}}<1.62 R_{\mathrm{N}}$, however, are so energetic that once Pluto leaves Neptune's sphere of influence, it is not bound to the Solar System. Only for $a_{\mathrm{P}}>$ $54.9 R_{\mathrm{N}}$ is Pluto definitely bound. The accepted mass of Triton, $1.320 .23 \times 10^{26} \mathrm{~g}$, is due to Alden ${ }^{2}$. The combined mass of Pluto and Charon is known more precisely $( \pm 5 \%$ ) (ref. 3 ) and suggests $m_{\mathrm{T}} / m_{\mathrm{P}} \sim 10$. In this case, momentum conservation alone determines that a Lyttleton-type interaction is not possible, unless the interaction takes place well outside the domain of regular satellites. This domain, $a \leq 60 R_{\mathrm{N}}$, is defined in analogy with the regular satellite systems of Jupiter, Saturn and Uranus. The outermost regular satellite in each system, Callisto, Iapetus and Oberon, orbits at 27, 59 and 23 units of its respective planetary radius.

Recent radiometric measurements ${ }^{4,18,19}$ call Triton's mass estimate into question. Lebofsky's $2 \sigma$ detection, in particular, yields a radius of $1,750 \pm 250 \mathrm{~km}$ (ref. 19). In lieu of accepting Triton as a heavy metal satellite, I assume a density appropriate to an ice-rock body of this size $\left(\sim 1.6 \mathrm{~cm}^{-3}\right)$ (ref. 20)-implying that $m_{\mathrm{T}} / m_{\mathrm{P}} \sim 3$. This lower mass ratio, however, does not make Lyttleton's hypothesis more physically acceptable. Note that neither the effect of a non-unity emissivity nor a surrounding atmosphere has been included in the radiometric modelling, and these would have competing effects on the derived radius. A better determination of Triton's mass is needed.

These arguments can be generalized to allow multiple encounters if the conservation of energy is accounted for. The encounters are assumed to be conservative, with the only restriction on Triton's final energy, $-G m_{\mathrm{N}} m_{\mathrm{T}} / 2 \tilde{a}_{\mathrm{T}}$, where $\tilde{a}_{\mathrm{T}}$ is Triton's final semimajor axis, being that it exceed Triton's present value. The horizontal curves in Fig. 2 are Pluto's final semilatus rectum, $p_{\mathrm{P}}=l_{\mathrm{P}}^{2} / G m_{\mathrm{N}} m_{\mathrm{P}}^{2}=a_{\mathrm{P}}\left|1-e_{\mathrm{P}}^{2}\right|$, where $e_{\mathrm{P}}$ is uto's final eccentricity, for various $m_{\mathrm{T}} / m_{\mathrm{P}}$. The vertical curves are Pluto's final semimajor axis, given by

$$
a_{\mathrm{P}}=a\left\{\frac{m_{\mathrm{T}}}{m_{\mathrm{P}}}\left(\frac{a}{\tilde{a}_{\mathrm{T}}}-1\right)-1\right\}^{-1}
$$

for $m_{\mathrm{T}} / m_{\mathrm{P}}=10$ and two representative values of $\tilde{a}_{\mathrm{T}}$. Ascending asymptotes (with increasing $a$ ) correspond to bound orbits. For ellipses, however, $p<a$, so only those $a_{\mathrm{P}} \geq p_{\mathrm{P}}$ correspond to possible orbits. In addition, the pericentre distance, $p_{\mathrm{P}} /\left(1+e_{\mathrm{P}}\right)$,

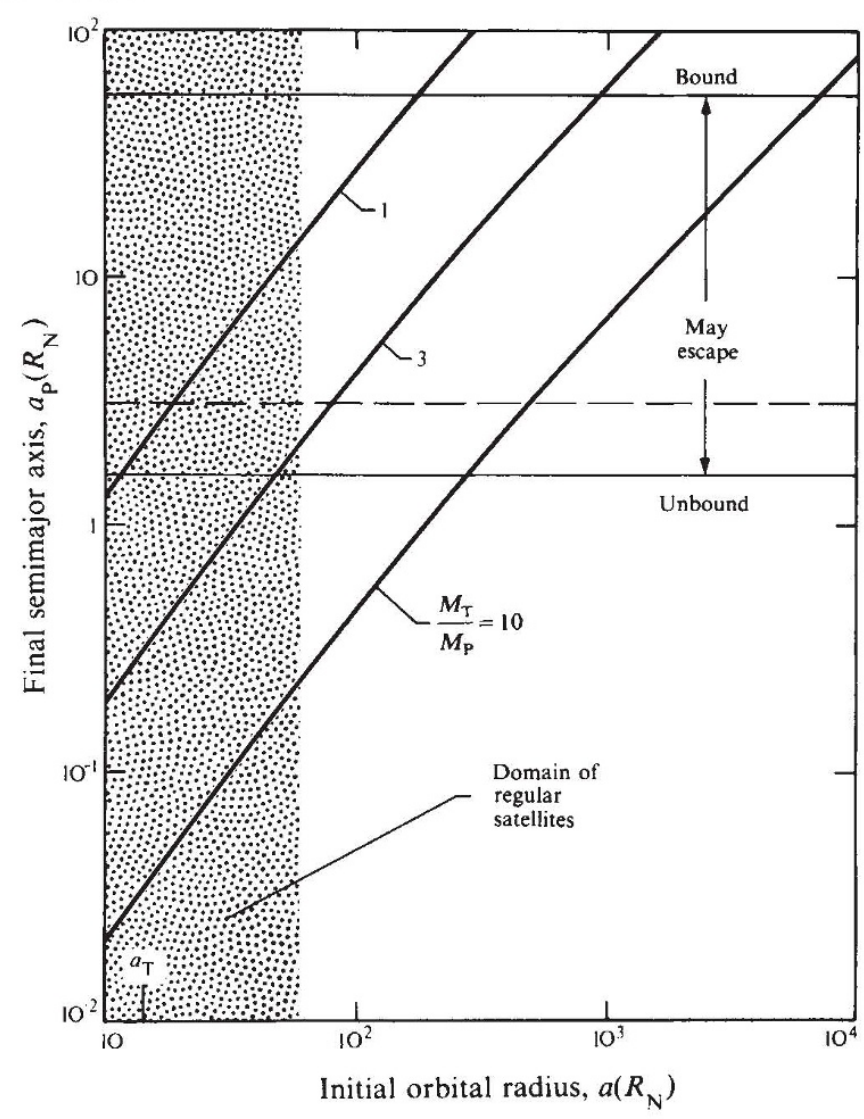

Fig. 1 Hyperbolic semimajor axis of Pluto, with respect to Neptune and after an interaction with Triton, as a function of initial orbital radius for three different mass ratios. These results, derived from angular momentum conservation alone, represent maximum values. Asymptotic velocities from Neptune of 2.25 and $13.1 \mathrm{~km} \mathrm{~s}^{-1}\left(a_{\mathrm{P}}=54.9\right.$ and $\left.1.62 R_{\mathrm{N}}\right)$ correspond to limits on bound or unbound solar trajectories, if Neptune's orbital velocity is taken into account. The dashed horizontal line is the minimum $a_{\mathrm{P}}$ for ejection into a direct, bound solar orbit. For plausible mass ratios, any interaction to reverse Triton's orbital motion and retain Pluto in the Solar System must take place well outside the domain of regular satellites (see text).

has a minimum of $p_{\mathrm{P}} / 2$ for $e_{\mathrm{P}}=1$, and Triton's apocentre distance has a maximum of $\sim 2 \tilde{a}_{\mathrm{T}}$. Thus even for $m_{\mathrm{T}} / m_{\mathrm{P}}=1$, no bound orbits are possible because the final orbits of Triton and Pluto must be $\sim$ intersecting ones. A descending branch of $a_{\mathrm{p}}$, for a given $\tilde{a}_{\mathrm{T}}$, is the locus of unbound states (with respect to Neptune). The top part of the descending branch $\left(a_{\mathrm{p}} \geq p_{\mathrm{p}}\right)$ corresponds to eccentricities of between $\sqrt{2}$ and 1 ; hence these orbits are also non-intersecting and are ruled out. The bottom of the descending branch corresponds to hyperbolic orbits with $e_{\mathrm{p}}>\sqrt{2}$; hence, for large enough $e_{\mathrm{P}}$, a pericentre distance small enough to allow intersecting orbits may appear feasible. The situation is very restricted, however, as $a_{\mathrm{p}}$ has a horizontal asymptote at $\tilde{a}_{\mathrm{T}} m_{\mathrm{P}} / m_{\mathrm{T}}$. This limits $e_{\mathrm{P}}$ to $\left\{p_{\mathrm{P}} / a_{\mathrm{P}}+1\right\}^{1 / 2}$. Pluto's final pericentre distance remains $>2 \tilde{a}_{\mathrm{T}}$ for $m_{\mathrm{T}} / m_{\mathrm{P}} \geq 1$, regardless of the choice of $\tilde{a}_{\mathrm{T}}$ or initial $a$. Thus, for plausible mass ratios, and under the stipulated initial conditions, a combination of orbital interactions to reverse Triton's motion cannot be found.

This conclusion, more severe than that due to momentum conservation alone, is not changed by considering tidal interaction with Neptune during the encounters. In addition, allowing the initial conditions to be set when the difference between the semimajor axes of the two 'satellites' closes to within the radius of either the sphere of influence, $s$, or an even wider sphere of perturbation, can bring Triton and Pluto's final orbits closer together (if Triton is the innermost of the pair), but not 


\begin{tabular}{|c|c|c|c|}
\hline & & Parameter values & \\
\hline Object & Quantity & Value & Reference \\
\hline Neptune & $\begin{array}{c}m_{\mathrm{N}} \\
\boldsymbol{R}_{\mathrm{N}} \\
\\
J_{2} \\
\text { Rotation } \\
\text { period }\end{array}$ & $\begin{array}{c}1.03 \times 10^{26} \mathrm{~kg} \\
24.75 \times 10^{3} \mathrm{~km} ; \\
1 \mathrm{bar}, \text { equatorial } \\
4.3 \times 10^{-3} \\
\sim 15 \mathrm{~h} ; \text { dynamical }\end{array}$ & $\begin{array}{l}36 \\
37 \\
17 \\
38\end{array}$ \\
\hline Uranus & $\begin{array}{c}\text { Mass } \\
\text { Radius } \\
\\
J_{2} \\
\text { Rotation } \\
\text { period }\end{array}$ & $\begin{array}{c}8.67 \times 10^{25} \mathrm{~kg} \\
25.5 \times 10^{3} \mathrm{~km} ; 1 \mathrm{bar}, \\
\text { equatorial } \\
3.35 \times 10^{-3} \\
15.5 \mathrm{~h} ; \text { dynamical }\end{array}$ & $\begin{array}{c}36 \\
39,40 \\
40 \\
40\end{array}$ \\
\hline Triton & $a_{\mathrm{T}}$ & $354.3 \times 10^{3} \mathrm{~km}$ & 17 \\
\hline Pluto & $\begin{array}{l}R_{\mathrm{P}} \\
Q_{\mathrm{P}} \\
k_{2 \mathrm{P}}\end{array}$ & $\begin{array}{c}\sim 1,500 \mathrm{~km} \\
\sim 100 \\
\sim 2.7 \times 10^{-2}\end{array}$ & $\begin{array}{c}41 \\
\text { Assumed } \\
\text { Based on unity } \\
\text { cgs density and } \\
\text { water-ice rigidity }\end{array}$ \\
\hline
\end{tabular}

sufficiently to have them interacting for $m_{\mathrm{T}} / m_{\mathrm{P}} \geq 1$. Of course, for $m_{\mathrm{T}} \ll m_{\mathrm{P}}$, Triton can be sent retrograde, but Pluto will remain direct.

The present angular momentum state of the Pluto-Charon system (that is, that contained in their mutual orbits and spins) is not consistent with a Neptune satellite origin either. If PlutoCharon ever orbited Neptune with a semimajor axis $a$, tides raised on Pluto by Neptune would extract angular momentum from the system at a rate

$$
\frac{\mathrm{d} H}{\mathrm{~d} t}=\frac{3}{2} \frac{G k_{2 \mathrm{P}} m_{\mathrm{N}}^{2} R_{\mathrm{P}}^{2}}{a^{6} Q_{\mathrm{P}}}
$$

where $H$ is the angular momentum, and $R_{\mathrm{p}}, Q_{\mathrm{P}}$ and $k_{2 \mathrm{P}}$ are, respectively, Pluto's radius, specific dissipation function and potential Love number (see Table 1). The present angular momentum of Pluto-Charon is uncertain due to the difficulty in estimating Charon's mass, but probably lies between 2.4 and $6.6 \times 10^{37} \mathrm{~g} \mathrm{~cm}^{2} \mathrm{~s}^{-1}$. Extraction of angular momentum would cause the Pluto-Charon system to contract and eventually coalesce. The coalesced object would then be spun down to synchronous rotation ${ }^{6}$. The time scale for 'fusion' and spin-down is

$$
\tau=H /\left(\frac{\mathrm{d} H}{\mathrm{~d} t}\right) \sim 1 \times 10^{5}\left(\frac{a}{a_{\mathrm{T}}}\right)^{6} \mathrm{yr}
$$

Thus collapse of the Pluto-Charon system and spin-down would occur rapidly unless Pluto-Charon orbited Neptune at a great distance $\left(\tau \geqslant 100 \mathrm{Myr}\right.$ for $\left.a>45 R_{\mathrm{N}}\right)$. These same arguments apply if the original state of Pluto-Charon was a coalesced, rapidly-rotating proto-Pluto (the angular momentum limits above correspond to primordial rotation periods between $\sim 7$ and $11 \mathrm{~h}$; see refs 21,22 ).

Only a physical collision could impart the requisite angular momentum to Pluto-Charon prior to its ejection from the Neptune system. A close pass to Pluto by a more massive object could exert an extremely large torque on Pluto but the time scale for the interaction would be severely limited. A grazing pass by an object with even the mass of the Earth would be restricted to last a few $10^{3} \mathrm{~s}$, and the angular momentum imparted, according to equation (5), would be only a few per cent of the present total. A larger transfer of angular momentum may be possible for the slowest encounters with a massive grazer, if tidally-induced fission ${ }^{23}$ occurs, but the restriction of slow encounters again requires Pluto to initially be in a distant orbit about Neptune.

With regard to ejection dynamics, the reduction of the interaction cross-section from a gravitational one to approximately one of collision makes 'rogue' planet hypotheses such as that of

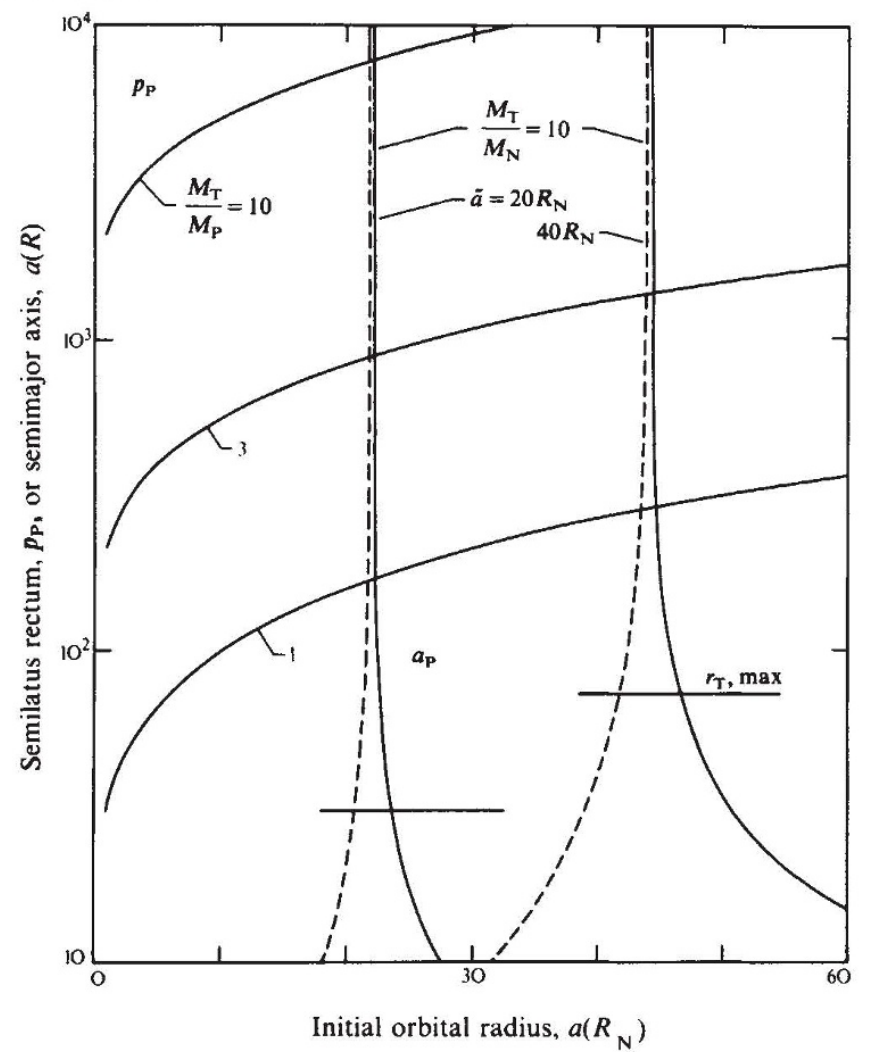

Fig. 2 Semilatus rectum and semimajor axis of Pluto, with respect to Neptune and after interactions with Triton, as a function of initial orbital radius for various mass ratios and final Triton semimajor axes. Only the solid part of the asymptotes represent possible orbits, but none have pericentre distances that approach Triton's final apocentre distance (short horizontal lines). Hence, for mass ratios greater than one, a series of interactions that reverses Triton's orbital motion and that conserve angular momentum and orbital energy cannot be found.

Harrington and Van Flandern ${ }^{24}$ probabilistically untenable. Farinella et al. call on a captured Triton to eject Pluto as its capture orbit decays ${ }^{25}$, but this hypothesis still requires a physical collision with Pluto, following which, gravitationally-bound plutonian debris accretes into a binary and eventually reaches stable, resonant ${ }^{26}$ solar orbit; the last requires an additional mechanism. This is an unlikely series of events.

The preceding arguments effectively decouple the origin of Pluto from that of any Neptune satellite. Hence, for Triton to begin as a direct satellite and end up retrograde would require interaction with an unknown and ad hoc object. I propose a simple reversal of Lyttleton's hypothesis: instead of beginning as satellites of Neptune, Triton and Pluto originate as satellites of the Sun. The existence of large, outer Solar System planetesimals is consistent with the dynamics of planetary accretion and may even be necessary to explain planetary eccentricities, inclinations, and obliquities (see ref. 27). PlutoCharon's binary status could be related to hypotheses regarding binary asteroid formation, ${ }^{21,28-30}$ and Triton's capture could be effected by the combination of a retrograde, temporary orbit about Neptune ${ }^{31}$ and a dissipative mechanism such as gas $\operatorname{drag}^{32,33}$.

If Triton had been captured, then it should have experienced a spectacular thermal event. As $Q_{N} \gg Q_{T}$, nearly all of the orbital energy dissipated during post-capture orbital evolution is deposited in the body of Triton. Gas drag, while potentially important for capture, did not result in a major change in orbital elements, as the evolution of Triton's inclination has been moderate $^{32}$. Calculations ${ }^{7}$ show that collapse of an elliptical postcapture orbit, extending to the edge of Neptune's sphere of influence $\left(\sim 3,500 R_{\mathrm{N}}\right)$, to one where the semimajor axis is 
within a few per cent of the present value, requires $\sim 4 Q_{\mathrm{T}} \mathrm{Myr}$, for a constant $Q_{\mathrm{T}}$. Most of the energy $(\sim 80 \%)$, however, is dissipated in an $\sim 1.5 Q_{\mathrm{T}} \mathrm{Myr}$ period near the end. The equivalent surface heat flow, for reasonable $Q_{\mathrm{T}}$, is far too great for solid-state convection in ice to transport, so it is extremely probable that the icy component of Triton completely melted during the latter phase of capture. $Q_{\mathrm{T}}$ itself would plummet as melting began, accelerating differentiation.

Received 27 April; accepted 24 July 1984.

1. Lyttleton, R. A. Mon. Not. R. astr. Soc. 97, 108-115 (1936).

2. Alden, H. L. Astr. J. 50, 110-111 (1943).

3. Harrington, R. S. \& Christy, J. W. Astr. J. 86, 442-443 (1981).

4. Cruikshank, D. P., Stockton, A., Dyck, H. M., Becklin, E. E. \& Macy, W. Jr Icarus 40 , 104-114 (1979).

5. McKinnon, W. B. Bull. Am. astr. Soc. 14, 765 (1982).

6. Lin, D. N. C. Mon. Not. R, astr. Soc. 197, 1081-1085 (1981).

7. McKinnon, W. B. Bull. Am. astr. Soc. 15, 857 (1983).

8. Cruikshank, D. P. \& Silvaggio, P. M. Astrophys. J. 223, 1016-1020 (1979).

9. Rieke, G. H., Lebofsky, L. A., Lebofsky, M. J. \& Montgomery, E. F. Nature 294, 59-60 (1981).

10. Apt, J., Carleton, N. P. \& Mackay, C. D. Astrophys. J. 270, 342-350 (1983).

11. Cruikshank, D. P., Brown, R. H. \& Clark, R. N. Abstr. Natural Satellites, IAU Colloq. No. 77,40 (1983).

12. Goldreich, P. \& Soter, S. Icarus 5, 375-389 (1966).

13. Munk, W. H. \& MacDonald, G. J. F. The Rotation of the Earth Ch. 5 (Cambridge University Press, 1975).

4. Reitsma, H. J., Hubbard, W. B., Lebofsky, L. A. \& Tholen, D. J. Science 215, 289-291 (1982) 15. Veillet, Ch. thesis, Univ. Paris (1983).

16. Tisserand, F. Traité de Mécanique Céleste Vol. 4, Ch. 12 (Gauthier-Villars, Paris, 1896).

17. Harris, A. W. Abstr. Natural Satellites, IAU Collog. 77, 41 (1983)

18. Morrison, D., Cruikshank, D. P. \& Brown, R. H. Nature 300, 425-427 (1982).

19. Lebofsky, L. A., Rieke, G. H., \& Lebofsky, M. J. Bull. Am. astr. Soc. 14, 766 (1982).
Catastrophic melting of Triton's ices should have released its inventory of clathrated gases to the surface, which for accretion outside a circumplanetary nebula would be $\mathrm{CH}_{4}, \mathrm{CO}$, and $\mathrm{N}_{2}$ (refs 34,35 ). A condensed fraction of this primordial atmosphere may be responsible for Triton's unique surface compositionpresent spectral identifications include liquid nitrogen ${ }^{11}$ and methane ice ${ }^{9-11}$.

This work was supported by NASA grant NAGW-432.

20. Lupo, M. J. \& Lewis, J. S. Icarus 40, 157-170 (1979).

21. Weidenschilling. S. J. Icarus 64, 124-126 (1981).

22. Chandrasekhar, S. Ellipsoidal Figures of Equilibrium Chs 5, 6 (Yale University Press, New Haven, 1969).

23. Aggarwal, H. R. \& Oberbeck, V. R. Astrophys. J. 191, 577-588 (1974).

24. Harrington R. S. \& Van Flandern T. C. Icarus 39, 131-136 (1979).

25. Farinella, P., Milani, A., Nobili, A. M. \& Valsecchi, G. B. Moon Planets 20, 415-421 (1979); Icarus 44, 810-812 (1980)

26. Nacozy, P.E. \& Diehl, R. E. Astr. J. 83, 522-530 (1978)

27. Harris, A. W. \& Ward, W. R. A. Rev. Earth planet. Sci. 10, 61-108 (1982).

28. Weidenschilling, S. J. Icarus 44, 807-809 (1980).

29. Farinella, P., Paolicchi, P. \& Zappala, V. Astr. Astrophys. 104, 159-165 (1981).

30. Harris, A. W. Bull. Am. astr. Soc. 15, 828 (1983).

31. Huang, T.-Y. \& Innanen, K. A. Astr. J. 88, 1537-1547 (1983)

32. Pollack, J. B., Burns, J. A. \& Tauber, M. E. Icarus 37, 587-611 (1979)

33. Nakazawa, K., Komuro, T. \& Hayashi, C. Moon Planets 28, 311-327 (1983).

34. Lewis, J. S. \& Prinn, R. G. Astrophys. J. 238, 357-364 (1980).

35. Prinn, R. G. \& Fegley, B. Astrophys. J. 249, 308-317 (1981).

36. Abstr. Natural Satellites, IAU Colloq. 77, 43 (1983).

37. Freeman, K. C. \& Lyngå, G. Astrophys. J. 160, 767-780 (1970).

38. Hubbard, W. B. et al. Astr. J. (submitted).

39. Elliot, J. L., Dunham, E., Mink, D. J. \& Churms, J. Astrophys. J. 236, 1026-1030 (1980).

40. Elliot, J. L. et al. Astr. J. 86, 444-455 (1981)

41. Hege, E. K. et al. Icarus 50, 72-81 (1982).

\section{X-ray haloes around supernova remnants}

\section{G. E. Morfill*, L. O’C Drury $\dagger \&$ B. Aschenbach*}

* Max-Planck-Institut für Physik und Astrophysik, Institut für extraterrestrische Physik, 8046 Garching, FRG

$\dagger$ Max-Planck-Institut für Kernphysik, 6900 Heidelberg, FRG

Recent observations of the Cas-A supernova remnant have shown $X$-ray emissions not only from the interior, but also from a fainter 'halo' extending beyond what is normally regarded as the outer boundary, or shock front ${ }^{1}$. We suggest here that this may be due to the diffusion of energetic, charged particles out of the remnant giving rise to precursor structure of the type predicted by the theory of diffusive shock acceleration. If this is the case we are seeing thermal emission from ambient gas heated by compression and wave dissipation.

The radio synchrotron emission from supernova remnants is clear evidence that they contain relativistic electrons; it is widely believed that they are also the major source for the galactic cosmic-ray nucleon component, but so far there has been no direct observational evidence for this. Cosmic-ray nuclei have an energy density which exceeds that of the electrons by about two orders of magnitude; for supernova remnants to be responsible for their origin, this implies that a substantial part of the remnant energy must reside in energetic charged particles ${ }^{2}$. This raises the question of how these particles are accelerated. Interstellar plasmas are typically so tenuous that cosmic rays are scattered only by the irregular structure of the magnetic field. Because the field lines are effectively frozen into the background plasma these irregularities can move relative to the bulk fluid at maximum speeds of the order of the magnetosonic speed. As Fermi $^{3}$ recognized, this is a rather unusual physical system in that macroscopic degrees of freedom, the magnetic fluctuations and their associated plasma, couple to a few individual particles. If the fluctuations are excited randomly this leads to a diffusion of the particles in momentum space which is the standard second-order Fermi acceleration mechanism. This is normally slow; however, at a shock front where the bulk plasma together with its embedded magnetic structures is suddenly decelerated (in the shock frame) the scatterers, instead of moving randomly, converge, giving rise to a much faster first-order acceleration ${ }^{4-8}$. Perhaps the major attraction of this process, usually called diffusive shock acceleration ${ }^{9-11}$, is that an elementary analysis in which the reaction of the particles is neglected, predicts that the accelerated particles should have power-law spectra in momentum with exponents close to those inferred for the sources of the galactic cosmic rays.

It is, however, clear that the reaction of the particles on the scattering irregularities, and through these on the bulk plasma flow, cannot be ignored if the energy density of the particles is significant, as is thought to be the case in supernova remnants ${ }^{5,12}$. The particles diffuse ahead of the shock into the upstream region and produce an adverse pressure gradient which decelerates the flow into the shock. This gradient also works on the scatterers at the rate $-v \cdot \operatorname{grad} P_{\mathrm{c}}$, where $v$ is the velocity of the scatterers and $P_{c}$ is the energetic particle pressure; clearly, and as one would intuitively expect, those fluctuation modes which move upstream relative to the plasma are amplified and those which move downstream are damped. The energy transferred to the amplified modes can be estimated to be of the order of the Alfvén Mach number of the shock times the background mag. netic field energy ${ }^{9,13}$. For strong astrophysical shocks, Alfvén Mach numbers of 20 or greater are expected. Thus in the absence of any dissipation, or radical change in the nature of the modes, the energy content of the fluctuations would become many times that of the background field; it seems doubtful that so much energy could be stored without various instabilities setting in which transfer excess energy to the plasma and keep the fluctuation amplitude of the order of unity.

Although the details of this process are very complicated, its main features have been described by a simple model ${ }^{13-15}$ in which only the energy and momentum fluxes associated with the thermal particles (treated as an ideal non-relativistic gas), the energetic particles (treated as an ideal relativistic gas) and the magnetic fluctuations (taken to be damped backwards-travelling Alfvén waves) are considered, while the shock structure is taken to be plane and steady.

We have calculated the shock structure and the Bremsstrahlung emissivity predicted by this model. Figure 1 shows an example of a shock with Alfvénic Mach number 20 and equal thermal particle, energetic particle and magnetic pressures far upstream. This high Mach number should be characteristic of young supernova remnant shocks. As can be seen, the shock 